\title{
La publicidad en el programa "En Surco la basura sirve" y su efecto socio-educativo en la recolección de residuos sólidos domiciliarios en el distrito de Santiago de Surco (2012-2013)
}

Recibido: 29 de agosto de 2016

Aceptado: 01 de febrero de 2017

Publicado: 27 de noviembre de 2017
Lilian Carranza Salanitro

lilian.carranza@usil.pe

Universidad San Ignacio de Loyola (Perú)

Resumen: Los limeños generan 8.000 toneladas de basura diarias, arrojando desechos sólidos, inorgánicos y no biodegradables que contaminan el medio ambiente, alterando negativamente las condiciones del hábitat y generando serios problemas en la salud, vida, economía y cultura. En este contexto se realiza la presente investigación, considerando la normatividad legal vigente sobre ecología y medio ambiente. El efecto en referencia es de carácter no formal, manifestado en el recuerdo y comprensión de la publicidad; clasificación de la fuente de origen de los residuos sólidos domiciliarios; identificación, grado de afinidad, posicionamiento y evaluación del programa "En Surco la basura sirve", y gestión pública de la Empresa Municipal de Santiago de Surco (EMUSSA). La metodología científica utilizada es cualitativa; los datos obtenidos y los resultados son verificables, constatándose que las instituciones municipales pueden liderar proyectos que posicionen en la ciudadanía el principio universal de reducir, reusar y reciclar.

Palabras clave: Desechos sólidos, desechos inorgánicos, desechos no biodegradables, publicidad, posicionamiento, programa "En Surco la basura sirve".

Abstract: People from Lima generate 8,000 tons of garbage a day; throwing solid, inorganic and non-biodegradable waste that pollute the environment, altering negatively their habitat conditions and generating serious problems in their health, life, economy and culture. In this context; the current investigation is carried out; considering the actual ecology and environment legal norm. The effect in reference has non-formal character; it is manifested in the memory and understanding of the advertisement; classification of 
the source of household solid waste; identification, degree of affinity, positioning, and evaluation of the program: "En Surco la basura sirve" and public management of The Empresa Municipal de Santiago de Surco (EMUSSA).

Key words: Solid waste, Inorganic waste, Non-biodegradable waste, Advertising, Positioning, "En Surco la basura sirve" Campaign.

\section{Introducción}

El ser humano obtiene insumos del medio que lo rodea y los consume, agotándolos rápidamente. Muchos de estos productos post-usados son desechos sólidos inorgánicos no biodegradables que contaminan el medio ambiente, alterando nocivamente las condiciones normales mediante agentes químicos, biológicos o físicos, que traen como resultado la alteración de condiciones físicas, sociales, económicas, entre otras. Los desechos sólidos inorgánicos están compuestos por residuos de cartones, papeles, plástico, vidrios, hojalata, textiles, etc. En algunos casos, pueden tomar miles de años en degradarse, convirtiéndose así en agentes contaminantes.

Según Brack (2010), la mayor contaminación no es generada por las empresas industriales y mineras, sino por los ciudadanos de a pie. El Ministerio del Medio Ambiente (MINAM) ha reconocido que el $70 \%$ de la basura recolectada a nivel nacional es llevada a los botaderos no autorizados o es quemada directamente, lo que propicia la emisión de dióxido de carbono $\left(\mathrm{CO}_{2}\right)$ y de gas metano, 21 veces más contaminante que el $\mathrm{CO}_{2}$ (Brack, 2010).

Sarria (2011) explica que Lima genera "8.000 toneladas de basura diariamente: el 33\% de la que se produce en el país. Es un volumen suficiente para llenar hasta el tope el nuevo Estadio Nacional cada dos días. Esta impresionante cantidad de residuos va a parar en un 78\% a los seis rellenos sanitarios que existen en la ciudad. El resto, casi 2.000 toneladas, termina en descampados, cauces de los ríos limeños, chancherías clandestinas o calles de la ciudad"

La basura es un problema sanitario complejo grave. Se han practicado alternativas de solución como menor consumo de productos y la implementación de la educación ambiental. En el caso peruano, se han planteado $a$ ) diseños de políticas gubernamentales y regionales, $b$ ) diseño e implementación de políticas municipales en relación con la basura, y c) diseño e implementación de políticas municipales en relación con el reciclaje, que es un proceso físico, químico o mecánico que adecúa una materia ya usada para obtener una nueva materia prima, vale decir, un producto nuevo.

El denominado "Plan Integral de Gestión Ambiental de Residuos Sólidos" (PIGARS) fue impulsado por diferentes municipales provinciales y distritales en el 2011, que priorizaba los procedimientos de reciclaje por unidad familiar. Sin embargo, según el informe anual de residuos sólidos municipales en el Perú, realizado por el MINAM (2011), sólo 82 de las 195 provincias peruanas cuentan con un PIGARS, es decir, sólo el 42\%. En el 2013, la 
Municipalidad Metropolitana de Lima contrató los servicios del consorcio integrado por la ONG Ciudad Saludable, Peru Waste Innovation e IPES para trabajar el mencionado plan, pero hasta la fecha no se aprecia la implementación de varias acciones allí sugeridas.

De manera aislada, la Municipalidad de Santiago de Surco (MSS) lanzó en el 2009 una campaña de sensibilización que consistió en la visita de escolares y vecinos a su planta de reciclaje; el distrito de Magdalena ha empadronado y formalizado a sus recicladores. Dos años después, dicha comuna constituyó la Empresa Municipal de Santiago de Surco (EMUSSA), cuya finalidad es el reciclado de residuos inertes, estableciendo el programa de "recolección de residuos sólidos domiciliarios", cuya difusión se realizó mediante la campaña publicitaria "En Surco la Basura Sirve".

La estrategia consistió en el manejo de un proceso de publicidad mixto, compuesto por dos etapas: una de lanzamiento y otra de mantenimiento. La etapa de lanzamiento estuvo constituida por publicidad difundida a través de medios publicitarios convencionales masivos (televisión, radio y prensa escrita) con el objetivo de llegar a una audiencia amplia y lograr el mayor número de impactos. En la etapa de mantenimiento, se utilizó la publicidad de vallas, publicidad "boca a boca", redes sociales y activaciones, con el objetivo socioeducativo de difundir y sensibilizar a los vecinos del distrito para que participen activamente en el programa.

A cada familia que está inscrita en el programa se le hace entrega de una bolsa naranja para que almacene en ella los residuos inorgánicos. Como incentivo por su participación reciben un "bono naranja", que consiste en un descuento anual de sus arbitrios en un promedio de S/. 13 (trece nuevos soles peruanos). Los representantes de la MSS informan a los vecinos sobre los horarios de recolección de las bolsas naranjas, que son recogidas una vez a la semana para ser transportadas a la planta piloto recicladora, donde son clasificadas y procesadas. En la actualidad, el 20\% de la población del distrito (20 000 familias, aproximadamente 80000 habitantes) participa en el proyecto. Aún queda saber por qué el $80 \%$ restante no lo hace.

\section{Objetivos}

\subsection{Objetivo general}

Analizar cómo es la publicidad en el programa "En Surco la basura sirve" y cuál es su efecto socioeducativo en la recolección de residuos sólidos domiciliarios en el distrito de Santiago de Surco, durante el período 2012-2013.

\subsection{Objetivos específicos}

- Identificar los medios y mensajes publicitarios que utiliza el programa "En Surco la basura sirve" para la recolección de residuos sólidos domiciliarios en el distrito de Santiago de Surco, durante el período 2012-2013. 
- Determinar el efecto socio-educativo no formal de la publicidad en el programa "En Surco la basura sirve" para la recolección de residuos sólidos domiciliarios en el distrito de Santiago de Surco, durante el período 2012-2013.

\section{Metodología}

\subsection{Tipo de investigación}

La presente investigación es explicativa, diacrónica, prospectiva, focalizada, aplicada, empírica, documental, descriptiva y cualitativa. Es explicativa, porque sustenta racionalmente relaciones causa-efecto; es diacrónica, por su alcance temporal, ya que estudia los fenómenos en cuanto proceso; es prospectiva, porque se refiere al presente con proyección al futuro; es focalizada porque solamente abarca el área el distrito de Santiago de Surco; es aplicada, porque busca contribuir a la solución de los problemas planteados; es empírica, porque se trabaja con hechos observados y experimentados de manera directa en la realidad concreta; y es documental porque tiene como fuentes directas a materiales bibliográficos de tipo escrito y virtual.

\subsection{Diseño de la investigación}

Es una investigación correlacional cuantitativa que se ve representada en el siguiente diagrama, en la medida que trata de establecer niveles cuantitativos de asociación significativa entre las variables:

Figura 1: Investigación correlacional.

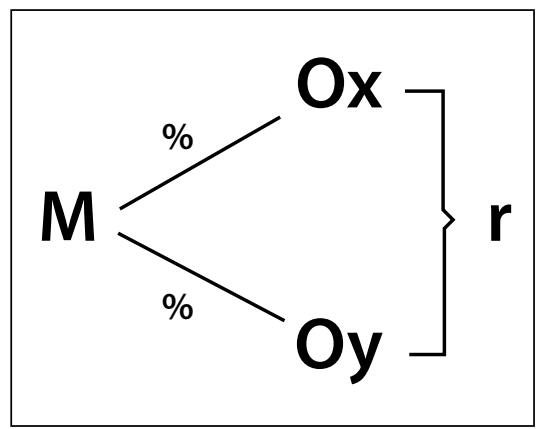

Fuente: Elaboración propia.

Donde:

$$
\begin{aligned}
\mathrm{M} & =\text { Muestra de investigación } \\
\text { Ox, Oy } & =\text { Observaciones de las variables } \\
\mathrm{R} & =\text { Relaciones entre variables }
\end{aligned}
$$




\subsection{Población y muestra}

La población del distrito de Santiago de Surco es de 321157 habitantes. La muestra aleatoria es de 160 .

\subsection{Operacionalización de variables}

\subsubsection{Variable independiente}

La publicidad del programa "En Surco la basura sirve".

Figura 2: Indicadores de la variable independiente.

\begin{tabular}{|c|c|}
\hline DIMENSIONES & INDICADORES \\
\hline $\begin{array}{l}\text { Tipos de anuncios } \\
\text { publicitarios }\end{array}$ & $\begin{array}{l}\text { - Nivel de recordación de difusión en televisión. } \\
\text { - Nivel de recordación de difusión de radio. } \\
\text { - Nivel de recordación de difusión de prensa. } \\
\text { - Nivel de recordación de campaña en redes sociales } \\
\text { (YouTube, Facebook y Twitter). } \\
\text { - Nivel de recordación de activaciones. } \\
\text { - Nivel de recordación de la comunicación boca a boca. } \\
\text { - Nivel de recordación de la marca EMUSSA en la campaña "En Surco la basura sirve". }\end{array}$ \\
\hline Mensaje publicitado & $\begin{array}{l}\text { - Nivel de recordación del mensaje. } \\
\text { - Nivel de comprensión del mensaje. } \\
\text { - Importancia adjudicada. }\end{array}$ \\
\hline Posicionamiento & $\begin{array}{l}\text { - Reconocimiento del proceso de clasificación de la fuente de origen en la selección } \\
\text { de los residuos. } \\
\text { - Grado de afinidad con el proceso de recolección de residuos sólidos domiciliarios. } \\
\text { - Forma cómo realizan la recolección de residuos sólidos domiciliarios. } \\
\text { - Aplicación de la etapa de clasificación de la fuente de origen. } \\
\text { - Posicionamiento de la frase "En Surco la basura sirve". } \\
\text { - Posicionamiento de la marca "EMUSSA". }\end{array}$ \\
\hline
\end{tabular}

Fuente: Elaboración propia. 


\subsubsection{Variable dependiente}

Efecto socioeducativo en la recolección de residuos sólidos domiciliarios.

Figura 3: Indicadores de la variable dependiente.

\begin{tabular}{|l|l|}
\hline DIMENSIONES & \multicolumn{1}{c|}{ INDICADORES } \\
\hline Percepción & $\begin{array}{l}\text { - Grado de afinidad con el proceso de recolección de residuos sólidos domiciliarios. } \\
\text { - Porcentaje de personas que realizan la recolección de residuos sólidos domiciliarios. } \\
\text { - Porcentaje de personas que realizan la etapa de clasificación de la fuente de origen. } \\
\text { - Efectividad de los puntos naranjas. } \\
\text { - Uso de las bolsas naranjas. }\end{array}$ \\
\hline \multirow{5}{*}{ Poblador de Surco } & $\begin{array}{l}\text { - Percepción que tienen del progra ma "En Surco la basura sirve". } \\
\text { - Atributos adjudicados. } \\
\text { - Concepto del reciclaje. Aspectos positivos y negativos. } \\
\text { - Perfil de la población que está realizando la campaña. } \\
\text { - Perfil de la población que no está realizando la campaña. } \\
\text { - Expectativas hacia el programa. }\end{array}$ \\
\hline
\end{tabular}

Fuente: Elaboración propia.

Las técnicas utilizadas para la recolección de datos fueron las siguientes: observación sistemática (con fichas codificadas de observación); entrevista formal, con cuestionario formal de entrevistas; libretas de campo y fichas codificadas de entrevista formal. También se aplicaron encuestas con cuestionarios impresos, grabaciones, transcripciones de audio, fotografías y fichaje de textos bibliográficos y virtuales confiables. Para el procesamiento y análisis de la información cualitativa se ha utilizado el software Atlas Ti. En lo que respecta a la data cuantitativa, se empleó el Excel, Stats y SPSS 20.

\section{Programa de segregación en la fuente y recolección selectiva de residuos sólidos domiciliario}

El distrito de Santiago de Surco es pionero en el manejo de residuos sólidos segregados, logrando ser referente en nuestro país en el desarrollo de las políticas de eliminación y recuperación de residuos sólidos con fines de recuperación del valor comercial residual del material. En junio de 1997 surgió el llamado "Programa de Segregación en Origen", junto con una gran campaña de Sensibilización vecinal denominada "En Surco la basura sirve", que buscaba concientizar a la población sobre la importancia de adoptar una posición responsable ante el significativo incremento en la generación de los residuos sólidos 
urbanos; contribuir con la preservación del medio ambiente; y mantener de una manera sostenible la calidad de vida de los vecinos.

Un equipo de promotores visitó, puerta a puerta, a los vecinos para proporcionarles información sobre la importancia ambien tal de separar los residuos reciclables en bolsas de color naranja que se les entregaría una vez a la semana. Los materiales reciclables que se colocan en éstas son plástico, vidrio, metal, papel y cartón. De esta manera, toda la familia participa y se incrementa el hábito de la separación de los residuos (Asencios, 2016).

En el programa participaron los vecinos, así como las instituciones comerciales y educativas, logrando un beneficio tanto social como económico y ambiental. Actualmente es operado por la empresa Municipal de Santiago de Surco S. A. (EMUSSSA), que realiza la captación de nuevos vecinos, capacitación, recojo diferenciado, segregación, clasificación y comercialización, a la vez que administra una planta de operaciones donde se producen 10 toneladas diarias de material reciclado.

Entre sus logros destacan la capacitación permanente a los vecinos, la participación de ocho sectores del distrito y la eliminación de 360 toneladas diarias de residuos sólidos. En los siguientes subapartados se enumeran los componentes técnicos.

\subsection{EMUSSA}

Es uno de los principales componentes operativos que soporta al programa, ya que a partir de las condiciones de operación actualmente existentes, se define el éxito de la gestión de residuos sólidos segregados. EMUSSA es una empresa pública con régimen privado, su optimización, mejora y planes de crecimiento no dependen de la municipalidad. Sin embargo, se estima que al finalizar el periodo 2012-2014 se logrará un incremento del $10 \%$ en la producción de materiales recuperados debido a mayores tasas de generación y reducción de pérdidas de residuos por incursión de segregadores diurnos (MSS, 2012).

\subsection{Recicladores formalizados}

Incluye la segregación en la fuente del sector 2 y próximamente en el sector 9 del distrito de Santiago de Surco. En su primera etapa estuvo destinada a la población que posee viviendas y condominios multifamiliares con superficie mayor a $150 \mathrm{~m}^{2}$, donde es posible el almacenamiento de residuos segregados por siete días como es el caso de la bolsa naranja. En el sector 2 se identificaron 21318 viviendas, de las cuales el 20\% podría participar del programa, estimándose en 11.7 toneladas diarias la cantidad de residuos sólidos producidos factibles de recuperar (la generación de basura per cápita del sector 2 es de $0.65 \mathrm{~kg}$ por habitante por día) y con recuperación efectiva de 2.34 toneladas por día. Se estima que esta cantidad potencial de residuos podría brindar sostén a un máximo de 15 familias asentadas solo en el sector 2. Posteriormente se ampliará la participación de los segregadores a condominios ubicados en los sectores 5, 7 y 8 (MSS, 2012). 


\subsection{Contenedores soterrados}

Se han iniciado procesos formales de implementación de sistemas de contenedores soterrados en el distrito, que sean compatibles con el estándar europeo, empezando por la instalación de contenedores mellizos para almacenar residuos sólidos comunes y reciclables en los sectores 2 y 7 del distrito. Esta tarea está implementada con la adecuada señalización y sensibilización de los usuarios que se encuentran en las zonas beneficiadas (MSS, 2012).

En base a los precios de mercado registrados por la venta de materiales a cargo de la empresa EMUSSSA, durante el año 2011, se determinó que los mayores ingresos provienen de la venta de cartón corrugado (17.90\%) y pet mixto (11.06\%), sumando entre ambos S/ 434824 . Los menores porcentajes corresponden al cobre (2.02\%) y bolsa film (transparente) $(2.23 \%)$, con S/ 6753 de ingresos. Durante el año 2011 el total de ingresos por residuos suma S/ 1 494142 (MSS, 2012). En la siguiente tabla (figura 4) se muestra la valorización potencial mensual de los residuos sólidos reaprovechables, que ascendería a S/. 494 641.70, sumando así S/. 5935700.38 de manera anual.

Figura 4: Valorización potencial mensual de los residuos sólidos reaprovechables.

\begin{tabular}{|c|c|c|c|c|c|}
\hline \multirow{3}{*}{$\begin{array}{l}\text { TIPO DE RESIDUOS } \\
\text { SÓLIDOS (A) }\end{array}$} & $\begin{array}{l}\text { COMPOSICIÓN } \\
\text { PORCENTUAL }\end{array}$ & $\begin{array}{l}\text { GENERACIÓN DERS } \\
\text { APROVECHABLES }\end{array}$ & $\begin{array}{l}\text { POTENCIAL DE } \\
\text { SEGREGACIÓN } \\
\text { EFECTIVA }\end{array}$ & $\begin{array}{l}\text { CANASTA DE } \\
\text { PRECIOS }\end{array}$ & $\begin{array}{l}\text { ESTIMACIÓN } \\
\text { DE INGRESOS }\end{array}$ \\
\hline & (B) & (C) & (D) $=C * 0.25$ & $\mathbf{E}$ & $F=D^{*} \mathbf{E}$ \\
\hline & $\%$ & Tn/mes & Tn/mes & S/ kg & S/ mes \\
\hline Papel blanco & 2.10 & 201.23 & 50.31 & 0.98 & 49301.03 \\
\hline Papel mixto & 4.19 & 402.46 & 100.61 & 0.38 & 38233.46 \\
\hline Periódico & 6.29 & 603.69 & 150.92 & 0.30 & 45276.46 \\
\hline Guias telefónicas & 1.40 & 134.15 & 33.54 & 0.20 & 6707.62 \\
\hline Cartón primera & 4.24 & 406.41 & 101.70 & 0.47 & 47799.75 \\
\hline Cartón segunda & 2.12 & 203.40 & 50.5 & 0.38 & 19323.30 \\
\hline Cartón estucado & 0.71 & 67.80 & 16.95 & 0.38 & 6441.10 \\
\hline Madera & 0.19 & 18.24 & 4.56 & 0.12 & 547.26 \\
\hline Plástico duro & 1.59 & 153.03 & 38.26 & 0.78 & 29840.84 \\
\hline Plástico cd & 0.09 & 8.50 & 2.13 & 0.87 & 1849.11 \\
\hline Pet mixto & 2.04 & 195.54 & 48.88 & 1.83 & 89458.76 \\
\hline Bolsa mixta baja & 0.95 & 91.16 & 22.79 & 0.92 & 20966.96 \\
\hline Bolsa mixta alta & 1.64 & 157.46 & 39.36 & 0.87 & 34247.42 \\
\hline Bolsa transparente & 0.78 & 74.59 & 18.65 & 0.45 & 8390.93 \\
\hline Bolsa mixta & 0.69 & 66.30 & 16.57 & 1.41 & 18895.13 \\
\hline Policarbonato & 0.44 & 42.51 & 10.63 & 1.61 & 17109.60 \\
\hline Polipropileno & 0.13 & 12.75 & 3.19 & 0.45 & 1434.66 \\
\hline PVC & 0.09 & 8.50 & 2.13 & 0.00 & 0.00 \\
\hline
\end{tabular}




\begin{tabular}{|l|c|c|c|c|c|}
\hline Etiquetas & 0.26 & 24.86 & 6.22 & 0.45 & 2796.98 \\
\hline Metal ferroso & 1.74 & 166.61 & 41.65 & 0.76 & 31655.30 \\
\hline Aluminio & 0.12 & 11.67 & 2.92 & 3.56 & 10383.31 \\
\hline Vidrio & 4.30 & 412.74 & 103.18 & 0.11 & 11350.26 \\
\hline Tetra pak & 0.65 & 61.94 & 15.49 & 0.17 & 2632.45 \\
\hline Tecnopor & 0.33 & 32.00 & 8.00 & 0.00 & 0.00 \\
\hline Total recuperable & 37.06 & & & & 494641.70 \\
\hline
\end{tabular}

Fuente: MSS (2012).

\section{A continuación, se describe la ruta de la cadena del reciclaje (figura 5), integrado por nueve etapas (MSS, 2012):}

Figura 5: Proceso de reciclaje por parte de la Municipalidad.

\begin{tabular}{|c|c|c|}
\hline $\begin{array}{l}\text { GENERACIÓN } \\
\text { DE RESIDUOS }\end{array}$ & \multicolumn{2}{|c|}{ El vecino genera residuos sólidos domiciliarios como producto de sus actividades cotidianas. } \\
\hline $\begin{array}{l}\text { SEGREGACIÓN } \\
\text { EN LA FUENTE }\end{array}$ & \multicolumn{2}{|c|}{$\begin{array}{l}\text { Consiste en la separación de residuos de acuerdo a su condición de residuo aprovechable del } \\
\text { eliminable. En esta etapa interviene el generador, por lo cual es importante inculcar, sensibilizar y } \\
\text { capacitar en el reconocimiento, segregación y acopio adecuado de los residuos de valor comercial. }\end{array}$} \\
\hline $\begin{array}{l}\text { RECOLECCIÓN } \\
\text { CONVENCIONAL }\end{array}$ & \multicolumn{2}{|c|}{$\begin{array}{l}\text { Los servicios de limpieza pública de la Municipalidad eliminan los residuos que han sido descartados } \\
\text { del proceso de segregación en la fuente. }\end{array}$} \\
\hline $\begin{array}{l}\text { RECOLECCIÓN } \\
\text { SELECTIVA }\end{array}$ & \multicolumn{2}{|c|}{$\begin{array}{l}\text { El personal destinado a labores de recojo de residuos selecciona los residuos previamente segregados, } \\
\text { retirándolos de las calles. Esta labor deberá ser regulada por la supervisión de campo, ya que se } \\
\text { considera como una operación sensible a tareas sub estándar, como la segregación de residuos no } \\
\text { clasificados (con apertura de bolsa), por lo cual se deberá establecer e implementar un reglamento de } \\
\text { operaciones para su desarrollo. }\end{array}$} \\
\hline $\begin{array}{l}\text { TRANSPORTE DE } \\
\text { SEGREGADOS }\end{array}$ & \multicolumn{2}{|c|}{$\begin{array}{l}\text { Consiste en el transporte de residuos en vehículos acondicionados que permitan una recolección } \\
\text { adecuada. En el caso de transporte de residuos por asociaciones de recicladores, es realizado con } \\
\text { vehículos menores autorizados, identificados y empadronados que guardan todas las medidas de } \\
\text { seguridad y se encuentren sujetos a fiscalización de parte de la municipalidad. }\end{array}$} \\
\hline \multirow{5}{*}{$\begin{array}{l}\text { MANEJO DE } \\
\text { RESIDUOS BOLSA } \\
\text { NARANJA }\end{array}$} & $\begin{array}{l}\text { Pesado de } \\
\text { residuos }\end{array}$ & $\begin{array}{l}\text { Mediante la báscula electrónica ubicada en las instalaciones de la base } \\
\text { Municipal; descarga de residuos en una faja donde se separan y abren las bolsas } \\
\text { naranjas. }\end{array}$ \\
\hline & $\begin{array}{l}\text { Reciclaje } \\
\text { naranja }\end{array}$ & $\begin{array}{l}\text { Consiste en la fabricación de bolsas naranjas a partir de materia prima } \\
\text { recuperada, este es un proceso continuo de fabricación de bolsas que cuenta con } \\
\text { máquina de extrusión, peletizado, termo formado y estampado, permitiendo el } \\
\text { reciclaje efectivo en el proceso de fabricación de bolsas para la entrega a los } \\
\text { vecinos como parte del programa. }\end{array}$ \\
\hline & $\begin{array}{l}\text { Clasificación de } \\
\text { residuos sólidos }\end{array}$ & $\begin{array}{l}\text { Son las labores de separación de materiales con el uso de una línea de } \\
\text { clasificación que facilita la exposición de los residuos segregados para su mejor } \\
\text { separación, y acopio temporal antes de proceder a su almacenamiento. }\end{array}$ \\
\hline & $\begin{array}{l}\text { Acondiciona- } \\
\text { miento }\end{array}$ & $\begin{array}{l}\text { Consiste en la formación de paquetes cúbicos mediante el uso de prensas } \\
\text { hidráulicas que comprimen el material, facilitando su manejo para el } \\
\text { almacenamiento y comercialización. }\end{array}$ \\
\hline & $\begin{array}{l}\text { Almacena- } \\
\text { miento }\end{array}$ & El residuo acondicionado es almacenado para facilitar su manejo. \\
\hline
\end{tabular}




\begin{tabular}{|l|l|l|}
\hline \multirow{1}{*}{$\begin{array}{l}\text { Rechazo } \\
\text { miento }\end{array}$} & $\begin{array}{l}\text { Los responsables realizan el acopio adecuadamente, con la finalidad } \\
\text { de evitar cortes, punzadas y demás daños ocasionados por la } \\
\text { manipulación de residuos sólidos. }\end{array}$ \\
\cline { 2 - 3 } $\begin{array}{l}\text { RESIDUOS POR } \\
\text { ASOCIACIONES DE } \\
\text { RECICLADORES }\end{array}$ & $\begin{array}{l}\text { Los residuos son clasificados retirando los que no son adecuados, } \\
\text { cor lo cual son sometidos a una segunda clasificación según el valor } \\
\text { comercial del residuo. }\end{array}$ \\
\cline { 2 - 4 } & $\begin{array}{l}\text { Transporte } \\
\text { de residuos }\end{array}$ & $\begin{array}{l}\text { Los recicladores deben contar con un sistema de acopio apropiado para } \\
\text { su almacenamiento temporal, hasta lograr cuotas de almacenamiento } \\
\text { que permitan la venta de cantidades moderadamente transportables. }\end{array}$ \\
\cline { 2 - 4 } & $\begin{array}{l}\text { Ce realiza con vehículos debidamente acondicionados, autorizados } \\
\text { para el manejo de residuos sólidos. }\end{array}$ \\
\hline $\begin{array}{l}\text { lización de } \\
\text { residuos } \\
\text { sólidos }\end{array}$ & $\begin{array}{l}\text { La comercialización tanto de parte de EMUSSSA como de las } \\
\text { asociaciones de recicladores solo puede ser llevada a cabo por } \\
\text { empresas autorizadas auditables por la autoridad de salud y con } \\
\text { medidas de seguridad necesarias para garantizar un adecuado manejo } \\
\text { de los residuos valorizados. }\end{array}$ \\
\cline { 2 - 3 } & $\begin{array}{l}\text { Reaprove- } \\
\text { chamiento de } \\
\text { las materias } \\
\text { primas }\end{array}$ & $\begin{array}{l}\text { Las materias primas solo se proveerán a empresas que utilicen } \\
\text { los materiales reciclables en la fabricación y/o transformación de } \\
\text { materiales en productos nuevos, no está permitido el segundo uso de } \\
\text { materiales generados. }\end{array}$ \\
\hline
\end{tabular}

Fuente: MSS (2012).

Dicho proceso lo podemos observar en el siguiente flujo (figura 6).

Figura 6: Proceso de reciclaje de los residuos sólidos domiciliarios en Surco.

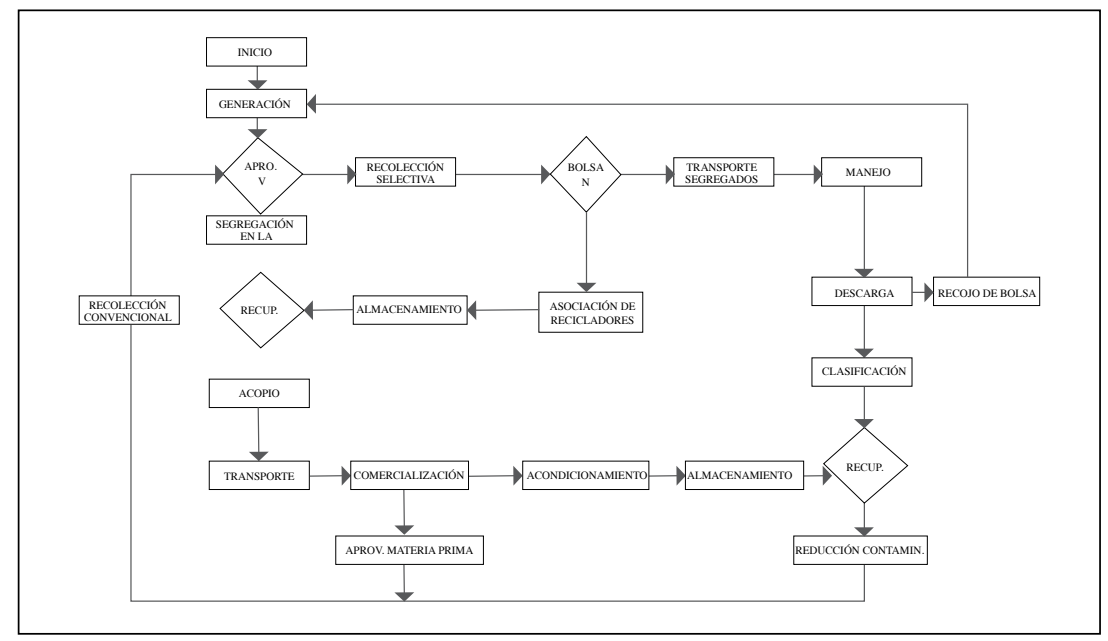

Fuente: MSS (2012).

Durante la campaña 2012-2013, se utilizó la publicidad social y diferentes estrategias mixtas para sensibilizar a la ciudadanía y fomentar la prevención, la reutilización y el reciclaje de residuos. En ese sentido, se enfatizó la participación de los vecinos de Surco en la recolección de residuos sólidos domiciliarios a través de la reciclable bolsa naranja, utilizando el slogan: "La bolsa que 
seguirá siendo bolsa". La gerencia de gestión ambiental distribuyó material divulgativo (folletos, bolsas y camisetas), emitió anuncios para reforzar conductas de consumo responsable en los distintos medios de comunicación y en las redes sociales (Facebook y Twitter).

Del mismo modo, la campaña estructuró un programa de educación ambiental sobre residuos y reciclaje, dirigido especialmente a la comunidad educativa. Así, se diseñaron personajes animados que hacían alusión a cada material para reciclaje ("Plasticón", "Papelín", "Vidrino" y "Latita") que aparecían en todas las piezas gráficas desarrolladas para la campaña. Una estrategia alternativa fue la creación del tour "Surco Verde", que organizó y ejecutó un plan de visitas concertadas para escolares y pobladores del distrito a la planta de reciclado de residuos.

Se difundieron publirreportajes a través de diversos medios de comunicación, tales como la televisión ("Haciendo Perú") y la web (portal Infoandina), así como en el diario El Comercio y en la revista Ecomundo. Los alcances del programa de reciclaje se transmitieron mediante paneles publicitarios, banners y grafitis. Asimismo, se distribuyeron volantes y afiches en las estaciones del punto naranja, con la intensión de motivar y captar más adeptos al programa. Se aplicó la publicidad directa en los domicilios de los ocho sectores del distrito de Santiago de Surco, enviándose afiches, folletos, artículos de merchandising y la bolsa reciclada naranja.

\section{Resultados y conclusiones}

La muestra aleatoria fue de 160 personas, Las técnicas utilizadas para la recolección de datos consistieron en la observación sistemática (con fichas codificadas de observación), la aplicación de una encuesta (con cuestionario formal de entrevistas de 28 preguntas) a los vecinos del distrito de Surco, la misma que se realizó en los días 7 y 9 del mes de noviembre de 2013.

Entre los medios de comunicación utilizados para colocar publicidad del programa, el q ue más ha impactado en los usuarios es el boca a boca (60\%), seguido de activaciones $(48.8 \%)$ y radio $(45 \%)$. Entre tanto, medios virtuales como Twitter $(0 \%)$, correo electrónico $(2.5 \%)$ y Facebook (3.8\%) han llegado a menos personas (figura 7).

Figura 7: Medios empleados durante la campaña "En Surco la basura sirve".

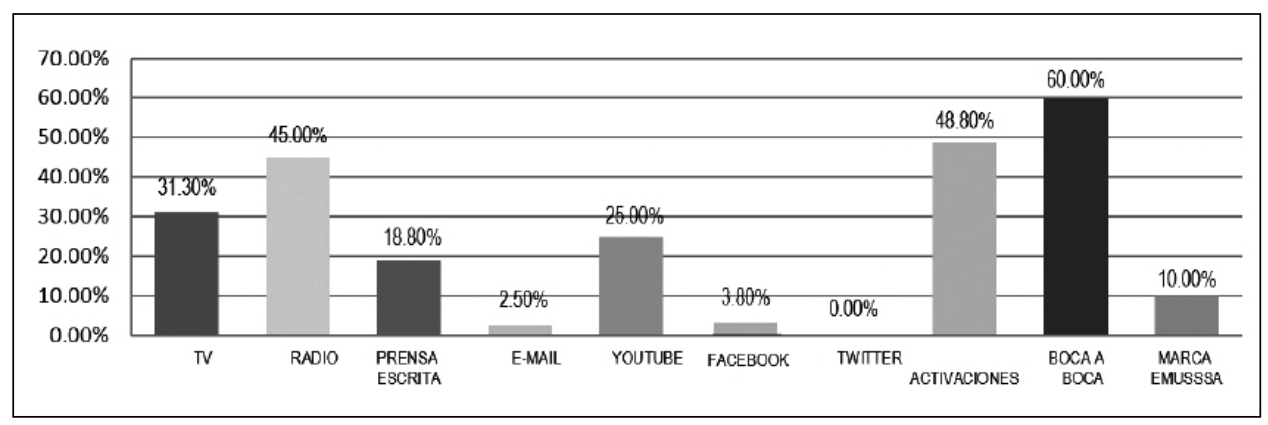

Fuente: Elaboración propia. 
Se observa que el e-mail es el medio que no genera recordación en el 98\% de la población, seguido de Facebook y Twitter, los cuales tampoco dejaron huella en el $96 \%$ de la muestra (figura 8). Solo el 10\% manifiesta que el medio "boca a boca" les posibilita mucho la retención de los mensajes, seguido de un $6 \%$ que también tiene en la memoria muchos de los mensajes a través de activaciones.

Figura 8: Nivel de recordación del programa "En Surco la basura sirve".

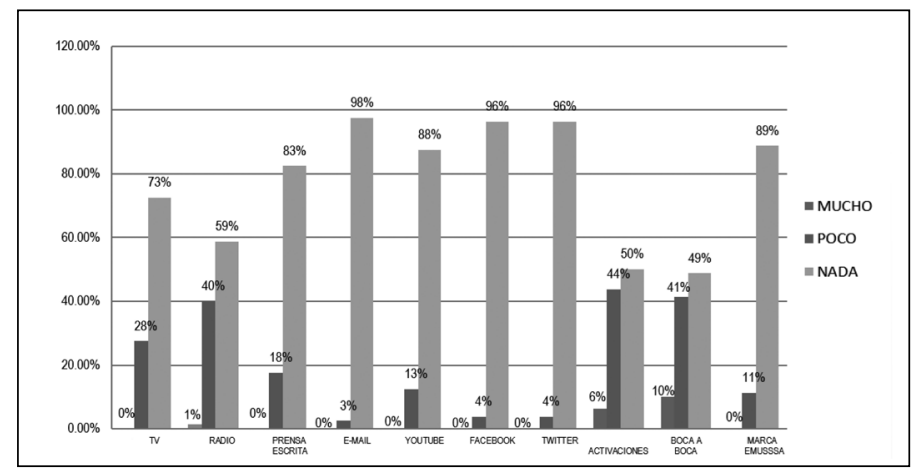

Fuente: Elaboración propia.

La publicidad cumple una función educativa no formal restringida a un sector poblacional; indicador de ella son los mensajes "En Surco la basura sirve" (47.5\%) y "Reciclar es tarea de todos" (35\%) que más recuerda este sector poblacional (figura 9).

Figura 9: Mensaje más recordado del programa "En Surco la basura sirve".

\begin{tabular}{|l|r|r|}
\hline \multicolumn{1}{|c|}{ MENSAJE } & F & \multicolumn{1}{c|}{$\%$} \\
\hline En Surco la basura sirve & 76 & $47.5 \%$ \\
\hline Reciclar es tarea de todos & 56 & $35.0 \%$ \\
\hline Ninguno & 28 & $\mathbf{1 7 . 5 \%}$ \\
\hline Total & $\mathbf{1 6 0}$ & $\mathbf{1 0 0 . 0} \%$ \\
\hline
\end{tabular}

Fuente: Elaboración propia.

El mensaje que más comprende la población en la publicidad del programa es "Mantener el ambiente limpio" (66.3\%), seguido del mensaje "Proteger el ambiente" (25\%). Se advierte que un $8.8 \%$ no comprende ningún mensaje (figura 10 ).

Figura 10: Mensaje más comprendido del programa "En Surco la basura sirve".

\begin{tabular}{|l|r|r|}
\hline \multicolumn{1}{|c|}{ MENSAJE } & F & \multicolumn{2}{c|}{$\%$} \\
\hline Mantener limpio el ambiente & 106 & $66.3 \%$ \\
\hline Proteger el ambiente & 40 & $25.0 \%$ \\
\hline Ninguno & 14 & $8.8 \%$ \\
\hline Total & $\mathbf{1 6 0}$ & $\mathbf{1 0 0 . 0} \%$ \\
\hline
\end{tabular}

Fuente: Elaboración propia. 
En relación con el posicionamiento de la publicidad del programa "En Surco la basura sirve" y su efecto socioeducativo en la recolección de residuos sólidos domiciliarios, se observa que el $52 \%$ sabe cuál es el proceso de clasificación de la fuente de origen en la selección de los residuos, pero el 48\% desconoce el proceso (figura 11).

Figura 11: ¿Sabe cuál es el proceso de clasificación de la fuente de origen en la selección de los residuos?

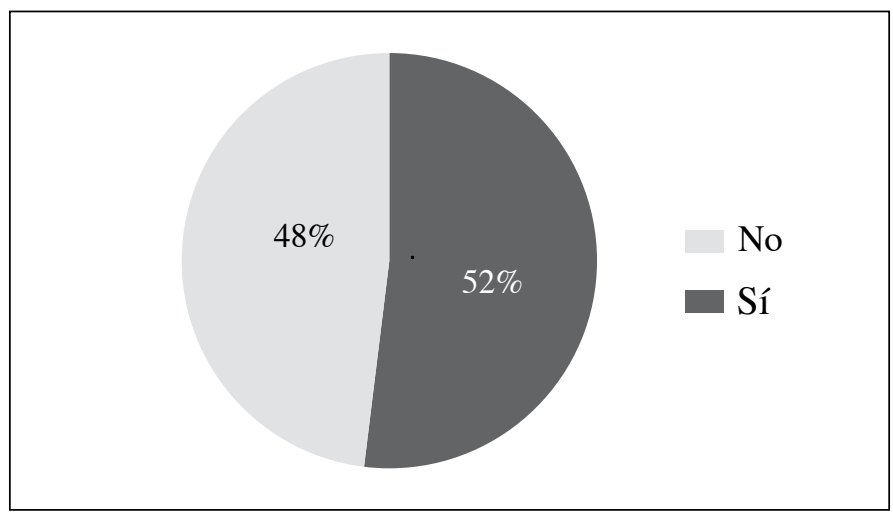

Fuente: Elaboración propia.

El 75\% está de acuerdo con el proceso de recolección de residuos sólidos domiciliarios, lo que debería influir en una mayor participación, preocupación y compromiso con el programa emprendido por el municipio (figura 12).

Figura 12: Grado de acuerdo o desacuerdo con el proceso de recolección de residuos sólidos domiciliarios.

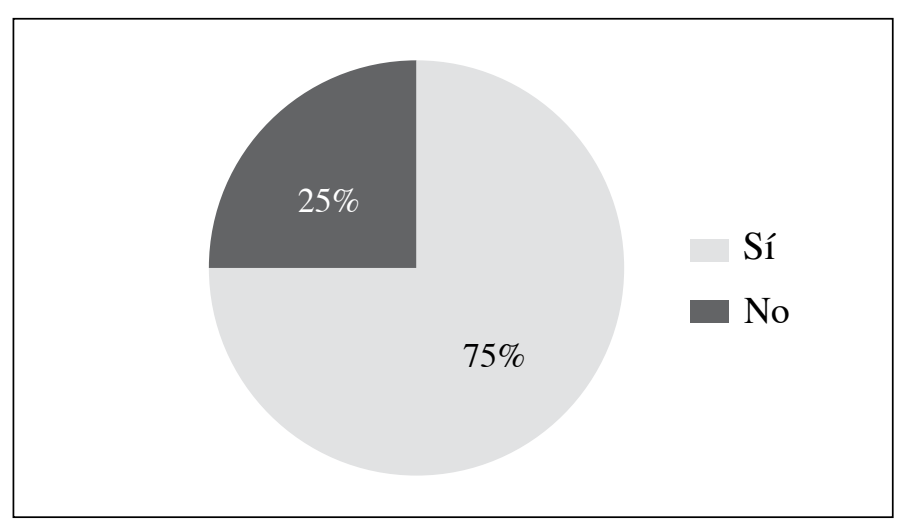

Fuente: Elaboración propia.

El 61\% desarrolla y ejecuta la etapa de clasificación de la fuente de origen, lo que demuestra su compromiso con el programa municipal (figura 13). 
Figura 13: Aplicación de la etapa de clasificación de la fuente de origen.

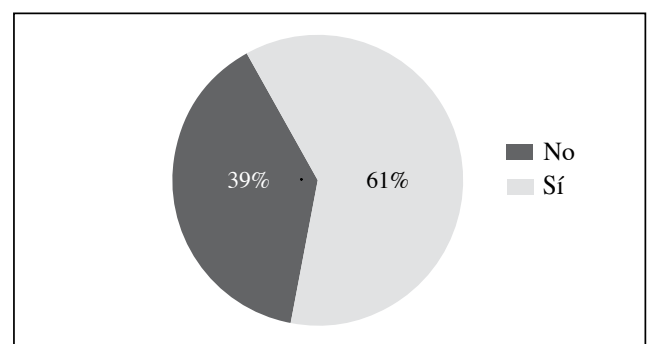

Fuente: Elaboración propia.

El $71.3 \%$ selecciona la basura y la deposita en una bolsa, el $23.8 \%$ selecciona la basura y clasifica en diferentes bolsas, siendo esta opción la óptima y la sugerida en la publicidad del programa. Sólo el 5\% no recolecta basura (figura 14).

Figura 14: Forma de recolección de residuos sólidos domiciliarios.

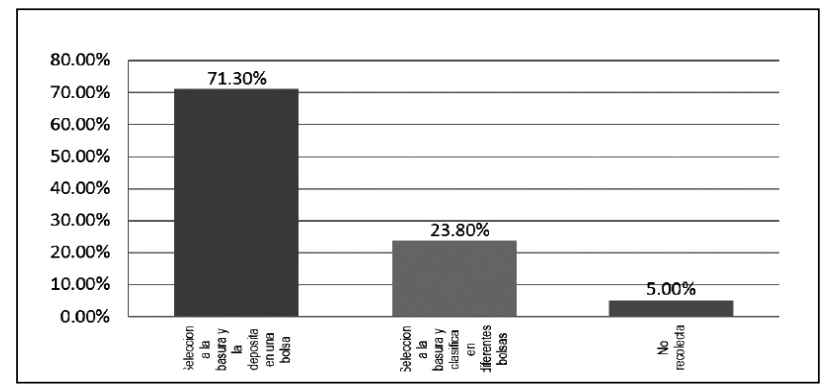

Fuente: Elaboración propia.

De estos resultados, se colige que el nivel de posicionamiento es intermedio, es decir, existe una limitada preocupación, compromiso y participación de la población en el programa. Por ello, el 61\% sí hace suya la frase "En Surco la basura sirve", hecho que denota también un grado medio de aceptación de la publicidad en el programa y su efecto socioeducativo en la recolección de residuos sólidos domiciliarios (figura 15).

Figura 15: Apropiación de la frase "En Surco la basura sirve".

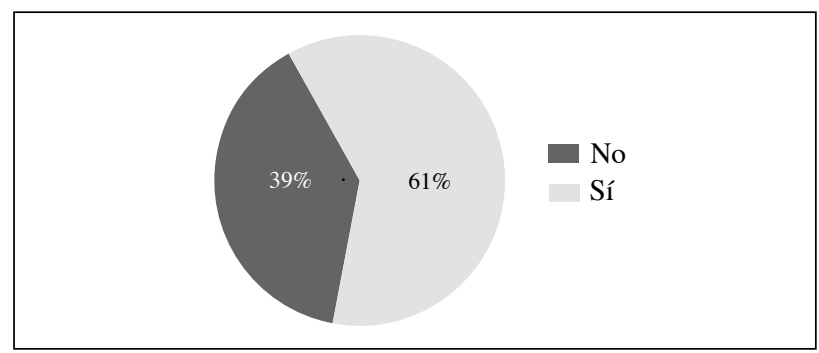

Fuente: Elaboración propia. 
El 62\% no se identifica con la marca EMUSSA, solo el 38\% lo hace. Esto último indica un bajo nivel de identificación con el primer y más importante componente de la publicidad del programa (figura 16).

Figura 16: Identificación con la marca EMUSSA.

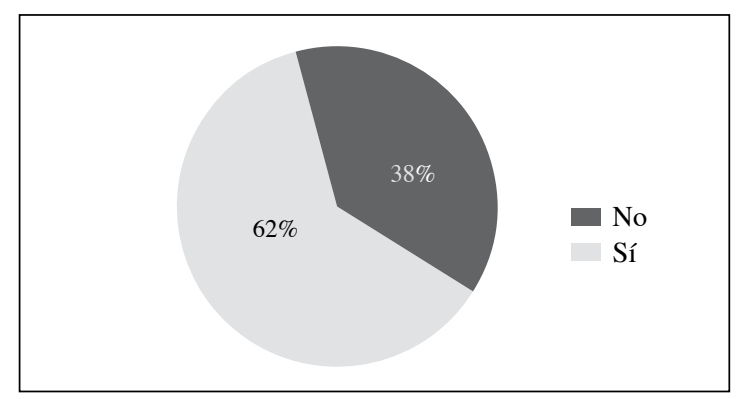

Fuente: Elaboración propia.

El 56.3\% no sabe con qué se identifica la marca EMUSSA; solo el $11.3 \%$ lo vincula con la planta de acopio de basura y el $32.5 \%$ con la planta de reciclaje de basura (figura 17).

Figura 17: ¿Con qué se identifica la marca EMUSSA?

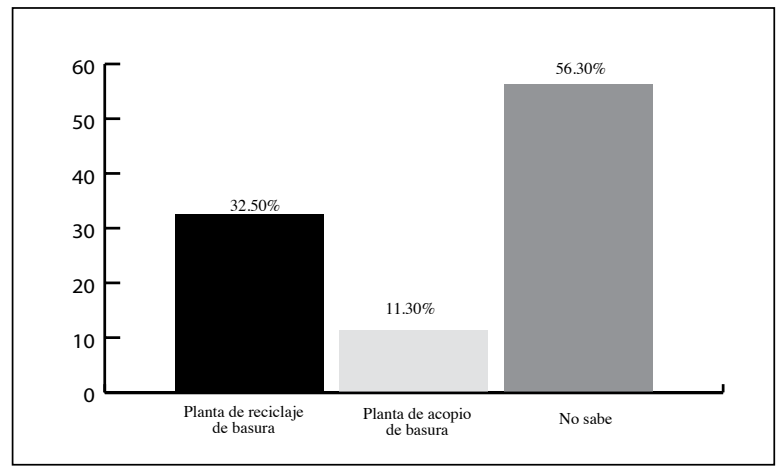

Fuente: Elaboración propia.

Con la publicidad del programa "En Surco la basura sirve" (y su efecto socioeducativo en la recolección de residuos sólidos domiciliarios) se logra parcialmente los objetivos relacionados con la dimensión persuasión: el 58.8\% piensa equivocadamente que reciclaje es "recolectar residuos para llevar a la planta", solo el $30 \%$ ofrece un concepto cercanamente acertado a lo que es reciclaje planteando que es "convertir residuos en materia prima" (figura 18). Se deduce que la mayoría de la población no tiene un concepto objetivo de reciclaje como proceso que reincorpora los residuos de consumo al circuito económico para valorizarlos, proceso físico, químico o mecánico que adecúa una materia ya usada para obtener una nueva materia prima, vale decir, un producto nuevo. 
Figura 18: Concepto de reciclaje.

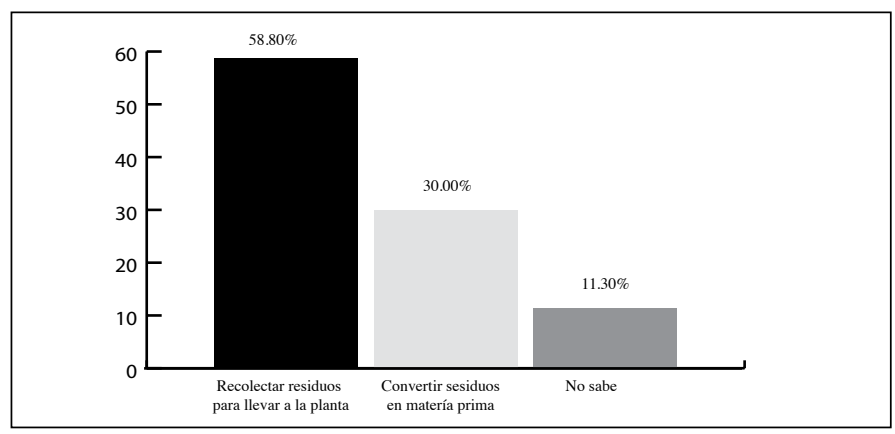

Fuente: Elaboración propia.

El 43\% tiene un grado medio de afinidad con el proceso de recolección de residuos sólidos domiciliarios. Otro porcentaje similar tiene un grado bajo de afinidad. Solo un $15 \%$ tiene un alto grado de afinidad (figura 19). Se deduce que el grado de afinidad con la publicidad del programa tiene una tendencia media.

Figura 19: Grado de afinidad con el proceso de recolección de residuos sólidos domiciliarios.

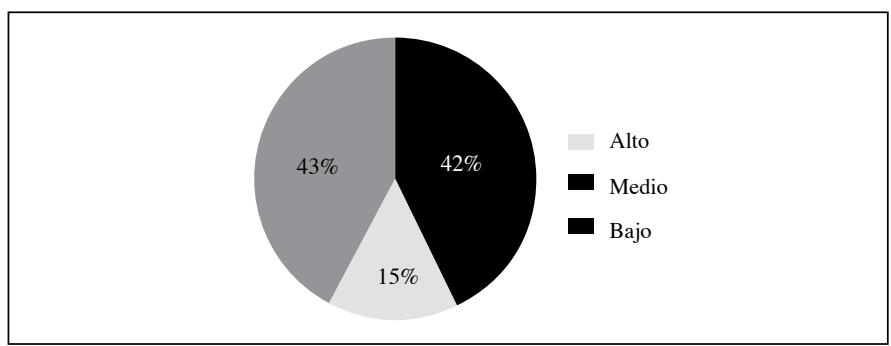

Fuente: Elaboración propia.

El 66\% no reconoce los puntos naranja, vale decir, no reconoce puntos de atención donde los vecinos pueden recabar las bolsas naranjas de reciclaje. Sólo el 34\% los reconoce (figura 20).

Figura 20: Efectividad de los puntos naranja.

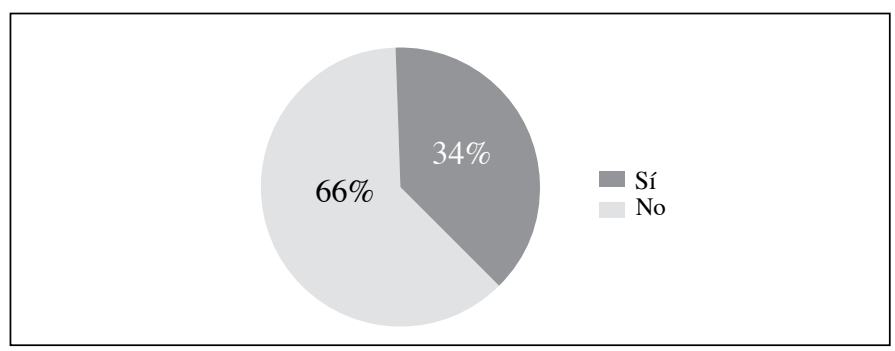

Fuente: Elaboración propia. 
Estos resultados develan que la persuasión por parte de la Municipalidad no es efectiva, lo cual se agrava con la realidad de que el $78 \%$ no utiliza la bolsa naranja (figura 21 ). Como ya se ha manifestado, esta bolsa es producto del material que se recicla en la propia planta de clasificación de residuos de la Municipalidad. La bolsa es el símbolo del programa y del reciclaje.

Figura 21: Uso de las bolsas de color naranja (BCN).

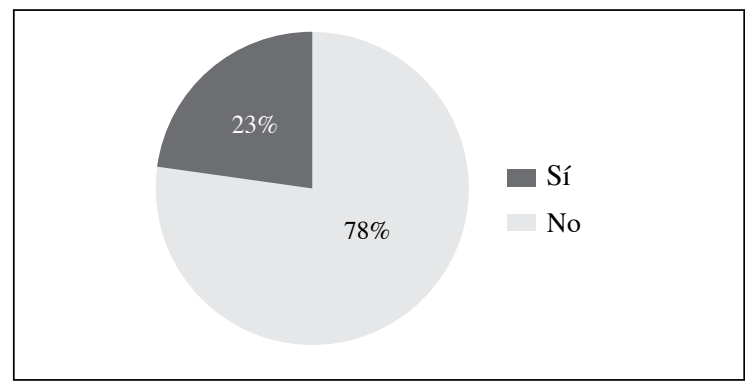

Fuente: Elaboración propia.

El grado de percepción acerca del programa es negativo para el 51\%. El 49\% tiene una percepción positiva (figura 22).

Figura 22: Grado de percepción del programa "En Surco la basura sirve”.

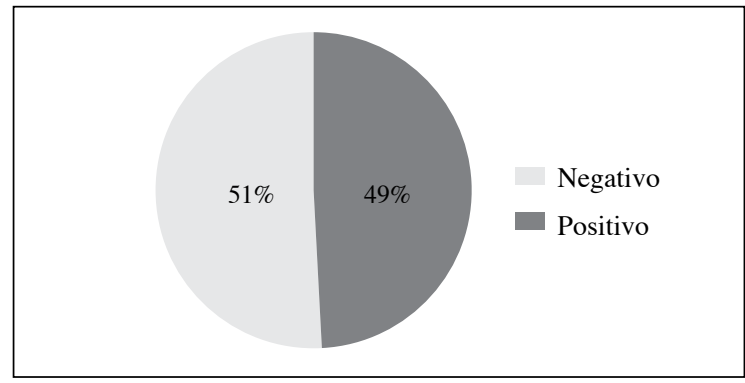

Fuente: Elaboración propia.

El 33.8\% evalúa como mala la gestión pública de EMUSSA, el 12.5\% piensa que es muy mala. El $32.5 \%$ la considera regular y solo el $16.3 \%$ la considera buena y el 5\% muy buena (figura 23 ). 
Figura 23: Grado de evaluación de la gestión pública de EMUSSSA.

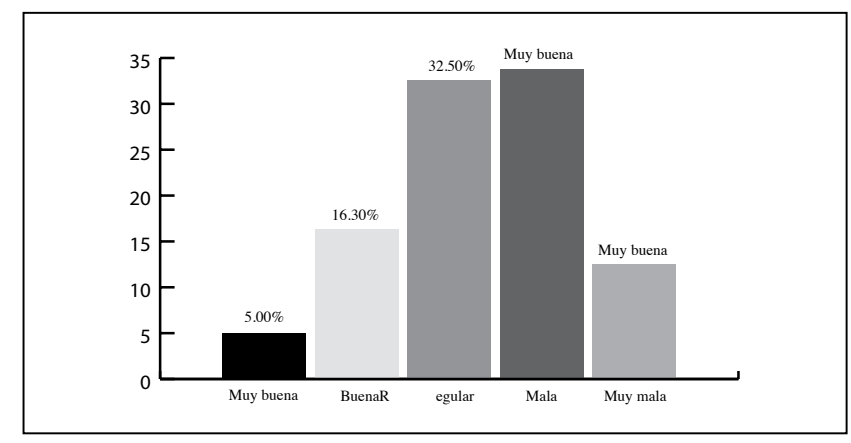

Fuente: Elaboración propia.

En suma, concluimos que la publicidad del programa "En Surco la basura sirve" y su efecto socioeducativo en la recolección de residuos sólidos domiciliarios contribuye parcialmente a la educación no formal en la recolección de residuos sólidos domiciliarios en los habitantes del distrito de Santiago de Surco.

\section{Fuentes consultadas}

Asencios, M. (2016). "Visita al parque ecológico Voces por el Clima". Extraída el 6/X/2017 desde http://parque-ecologico-voces-por-el-clima.blogspot.pe/2016/

Brack, A. (2010). " $83 \%$ de basura se va al ambiente". Extraída el 5/X/2017 desde http:// peru21.pe/noticia/466127/peru-83-basura-se-va-al-ambiente. 23-04-10

Ciudad Saludable (2013). "Inician talleres interdistritales descentralizados para el PIGARS Lima”. Extraída el 5/X/2017 desde http://www.ciudadsaludable.org/gabinete-decomunicacion/ultimas-noticias/item/61-inician-talleres-interdistritales-descentralizadospara-el-pigars-lima.html

MINAM, Ministerio del Medio Ambiente del Perú (2011). "Informe Anual de Residuos Sólidos Municipales en el Perú”. Extraída el 5/X/2017 desde http://sinia.minam.gob.pe/ download/file/fid/39726

MSS, Municipalidad de Santiago de Surco (2012). "Programa de Segregación en la Fuente y Recolección Selectiva de Residuos Sólidos Domiciliarios”. Extraída el 6/X/2017 desde http:/www.munisurco.gob.pe/municipio/laGestion/lasNormasEmitidas/ decretos/2012/DA\%2020-2012-MSS.pdf

Sarria, C. (2011). "Minam buscará fomentar en Limeños la cultura de reciclaje". Extraída el 5/X/2011 desde http://archivo.elcomercio.pe/ciencias/planeta/minam-buscara-fomentarlimenos-cultura-reciclaje-noticia-697948?ref=flujo_tags_523253\&ft=nota_112\&e=imagen 